IMA Journal of Applied Mathematics (2017) 82, 561-578

doi:10.1093/imamat/hxx003

Advance Access Publication on 27 February 2017

\title{
Birth of limit cycles from a 3D triangular center of a piecewise smooth vector field
}

\author{
Tiago Carvalho* \\ Departamento de Matemática, Faculdade de Ciências, UNESP, Av. Eng. Luiz Edmundo Carrijo Coube \\ 14-01, CEP 17033-360, Bauru, SP, Brazil. \\ *Corresponding author: tcarvalho@fc.unesp.br \\ RODRIGO D. EUZÉBIO \\ Departamento de Matemática, Universidade Federal de Goiás, IME, CEP 74001-970, \\ Caixa Postal 131, Goiânia, Goiás, Brazil \\ email: euzebio@ime.unicamp.br \\ Marco Antonto TeIXeira \\ Departamento de Matemática, IMECC-UNICAMP, CEP 13083-970, Campinas, SP, Brazil \\ email: teixeira@ime.unicamp.br \\ AND \\ DURVAL JOSÉ TONON \\ Departamento de Matemática, Universidade Federal de Goiás, IME, CEP 74001-970, \\ Caixa Postal 131, Goiânia, Goiás, Brazil \\ email: djtonon@ufg.br
}

[Received on 22 December 2015; revised on 18 October 2016; accepted on 27 January 2017]

\begin{abstract}
We consider a piecewise smooth vector field in $\mathbb{R}^{3}$, where the switching set is on an algebraic variety expressed as the zero of a Morse function. We depart from a model described by piecewise constant vector fields with a non-usual center that is constant on the sliding region. Given a positive integer $k$, we produce suitable nonlinear small perturbations of the previous model and we obtain piecewise smooth vector fields having exactly $k$ hyperbolic limit cycles instead of the center. Moreover, we also obtain suitable nonlinear small perturbations of the first model and piecewise smooth vector fields having a unique limit cycle of multiplicity $k$ instead of the center. As consequence, the initial model has codimension infinity. Some aspects of asymptotical stability of such system are also addressed in this article.
\end{abstract}

Keywords: periodic solutions; limit cycles; bifurcation; piecewise smooth vector fields

\section{Introduction}

\subsection{Setting the problem}

Going a step beyond the classical theory of topological dynamical systems, the theory of the so called piecewise smooth vector fields (PSVFs, for short) has been used to model a large number of phenomena. Indeed, both applications and theory of such a brand new area has been widely explored recently. Applications of piecewise smooth vector fields include but not limited to physics, control systems, electrical engineering and problems involving impact, among others. Some landmarks we can quote of such a theory are the work of Teixeira concerning manifolds with boundary (see Teixeira, 1977) and the book of Filippov, see Filippov (1988). One can also see the works of Broucke et al. (2001);

() The authors 2017. Published by Oxford University Press on behalf of the Institute of Mathematics and its Applications. All rights reserved. 


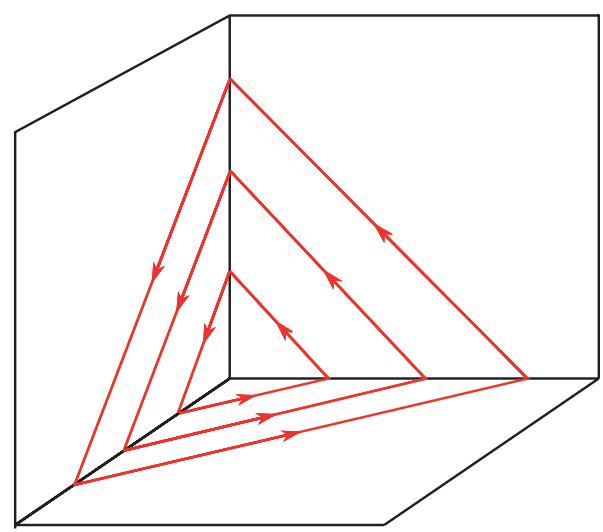

FIG. 1. The center contained in the switching manifold.

Chillingworth (2002); di Bernardo et al. (2008); Jacquemard et al. (2012); Kozlova (1984); Luo (2006); Teixeira $(1990,2008)$.

Basically, the difference between the classical theory concerning smooth dynamics and our approach lies in the fact that the late assume the existence of a codimension one variety $\Sigma$ separating the phase portrait in two or more regions, being defined in each region a distinct vector field.

In Filippov (1988), Filippov provided a convention to induce a vector field even on $\Sigma$, which is usually called 'Filippov (or sliding)' vector field. Generally, the first articles addressing PSVFs dealt with a variety $\Sigma$ generated by the preimage of a regular value of some smooth function. Meanwhile such a theory is enhanced by a huge range of applications, even when the discontinuity set is an algebraic variety. Models of PSVFs having $\Sigma$ in such a shape can be found in books of control or in articles taking into account relay systems (see, for instance, the book of Barbashin, 1970).

In this article, we analyse some qualitative aspects of a PSVF in $\mathbb{R}^{3}$ whose flow becomes confined on the 'switching manifold' $\Sigma$ after a finite time. Indeed, while in dimension 2 the dynamics on $\Sigma$ does not present any tricky behaviour, in dimension 3 one may observe some very interesting phenomena. In the present work, the switching manifold is formed by 12 parts (semi-planes), which give rise to eight different vector fields, defined in eight octants of $\mathbb{R}^{3}$.

In this scenario, we depart from a piecewise constant model with a non-usual center (see Fig. 1) that is constant on the sliding regions. So, we perform perturbations in order to study the existence of minimal sets as well as aspects concerning asymptotical and structural stability. Related articles that treat about the asymptotical stability in the context of PSVF are Teixeira (1990) and Jacquemard et al. (2013), for example.

We should stress that while we present an abstract model in order to study some aspects of PSVFs having a tricky switching manifold, the model we present is inspired in a formalism that is known in the literature as 'complementarity problem' (CP) (see Stewart, 2011 and references therein), which has a strongly connection with PSVFs and its importance in applications has been largely explored. More precisely, our approach in this work has some resemblance with the setting presented in Bernard and el Kharroubi (1991) and briefly discussed in Stewart (2011) (see pages 161-166). Observe that complementarity problems as DVIs (differential variational inequalities) or DCIs (differential complementarity problem) seems to be very important in describing physical situations presenting mechanical impacts or Coulomb friction. The connection between both, piecewise smooth's formalism and complementarity 
has been reported in the books of Acary and Brogliato Acary \& Brogliato (2008) (Chapters 1 and 2), di Bernardo et al. (2008) (Section 2.3.1) and Stewart (2011) (Chapter 1).

\subsection{Statements of the main results}

Consider the PSVF $Z_{0}$ defined in $\mathbb{R}^{3}$ by

$$
Z_{0}(x, y, z)=X_{i}(x, y, z), \text { if }(x, y, z) \in R_{i}, i=1,2, \ldots, 8,
$$

where each region $R_{i}, i=1,2, \ldots, 8$ is determined according to the following table:

\begin{tabular}{|l|c|c|c|c|c|c|c|c|}
\hline & $R_{1}$ & $R_{2}$ & $R_{3}$ & $R_{4}$ & $R_{5}$ & $R_{6}$ & $R_{7}$ & $R_{8}$ \\
\hline sign of $x$ & $\geq 0$ & $\leq 0$ & $\leq 0$ & $\geq 0$ & $\geq 0$ & $\leq 0$ & $\leq 0$ & $\geq 0$ \\
sign of $y$ & $\geq 0$ & $\geq 0$ & $\leq 0$ & $\leq 0$ & $\geq 0$ & $\geq 0$ & $\leq 0$ & $\leq 0$ \\
sign of $z$ & $\geq 0$ & $\geq 0$ & $\geq 0$ & $\geq 0$ & $\leq 0$ & $\leq 0$ & $\leq 0$ & $\leq 0$ \\
\hline
\end{tabular}

By considering a coherent arrangement of the regions listed previously, we get the switching region $\Sigma=\bigcup_{\{j, k\} \in I} \Sigma_{j, k}$, where $\Sigma_{j, k}$ is the boundary separating two adjacent regions $R_{j}$ and $R_{k}$, for $j, k \in I=$ $\{1,2, \ldots, 8\}$. We call $\Omega^{r}$ the space of vector fields $Z: \mathbb{R}^{3} \rightarrow \mathbb{R}^{3}$ having the form of (1.1) and observe that on $\Sigma$ we have defined two or more vector fields, in this case the classical Filippov's convention does not apply. Two approaches taking into account piecewise smooth systems having more than two vector fields defined on can be found in Dieci \& Lopez (2011) and Llibre (2015).

In our model, we consider the following choice of constant (linear) vector fields $X_{i}, i=1,2, \ldots, 8$ :

$$
\begin{aligned}
& X_{1}(x, y, z)=(-1,-1,-1), \\
& X_{2}(x, y, z)=(1,-1,3), \\
& X_{3}(x, y, z)=(1,-1,1), \\
& X_{4}(x, y, z)=(3,1,-1), \\
& X_{5}(x, y, z)=(-1,3,1), \\
& X_{6}(x, y, z)=(-1,1,1), \\
& X_{7}(x, y, z)=(1,1,1), \\
& X_{8}(x, y, z)=(1,1,-1) .
\end{aligned}
$$

In what follows, we write

$$
S=\Sigma_{1,2} \cup \Sigma_{1,4} \cup \Sigma_{1,5}
$$

Latter on, we show that $S$ is the sliding region of $\Sigma$ and it is a global attractor of System (1.1) (see Lemmas A and B, respectively).

Now we state the main results of the article.

THEOREM A. Let $Z_{0} \in \Omega^{r}$ be given by (1.1). For each integer $k \geq 0$, there exists an one-parameter family $Z_{\varepsilon} \in \Omega^{r}$ satisfying: 
(a) $Z_{\varepsilon} \rightarrow Z_{0}$ when $\varepsilon \rightarrow 0$;

(b) Restricted to $S, Z_{\varepsilon}$ has exactly $k$ hyperbolic limit cycles in a neighbourhood of the origin. The same holds for $k=\infty$ and,

(c) $Z_{0}$ possesses the invariant subset $S \subset \Sigma$ as a global attractor.

In order to announce the next result, let us roughly define the classical notion of codimension of vector fields.

DEFINITION 1 Consider $\Theta(W)$ the set of all small perturbations of a vector field $W$, defined on a compact set $K$. We say that $W$ has codimension $k$ if it appears exactly $k$ distinct topological types of vector fields in $\Theta(W)$.

An immediate consequence of Theorem A is:

Corollary B. The PSVF $Z_{0} \in \Omega^{r}$, given by (1.1), has infinite codimension.

Moreover, the next theorem brightens the scenario around $Z_{0}$.

In the next result, consider $\Omega^{r}(K)$ the space of the PSVF, with domain restricted to a compact subset $K \subset \mathbb{R}^{3}$ around the origin.

TheOREM C. Let $Z_{0} \in \Omega^{r}(K)$ be given by (1.1). For each neighbourhood $\mathcal{V}$ of $Z_{0}$ and for each integer $k>0$ there exists $Z_{\varepsilon}^{k} \in \mathcal{V}$ having codimension $k$.

Next result also provides informations about the asymptotical stability of the origin.

THEOREM D. There exist $Z_{\varepsilon}^{0} \in \Omega^{r}$, an one-parameter family of perturbations of the PSVF $Z_{0}$, for which the origin is either asymptotically stable or unstable, depending on the sign of $\varepsilon$.

Theorems A and C and Corollary B are proved in Section 5. Theorem D is proved in Section 3.

The article is organized as follows. In Section 2, we introduce the terminology, some definitions and the basic theory about PSVFs. In Section 3, we study the main properties of System $Z_{0}$. In Section 4, suitable perturbations of this system are considered. Moreover, the birth of limit cycles are explicitly exhibited. In Section 5, we prove the main results of the article. Finally, in Section 6, we present a brief conclusion.

\section{Preliminaries}

Inspired in the models presented in Bernard and el Kharroubi (1991) (see also Stewart, 2011) we first consider the particular PSVF $Z_{0}$ given by (1.1). At the sequel, we formalize some theory about this kind of vector field.

Denote $\pi_{i}: \mathbb{R}^{3} \longrightarrow \mathbb{R}$ the projection onto the $i$-th coordinate, $i=1,2,3$ and observe that

$$
\Sigma=\bigcup_{i=1,2,3} \pi_{i}^{-1}(0)
$$


We remark that once $\Sigma$ is not regular, the coordinate planes $A_{i}=\pi_{i}^{-1}(0)$ itself, $i=1,2,3$, do not play any special role in our approach. However, they allows us to define the function $h: \mathbb{R}^{3} \backslash \mathcal{F}_{1} \longrightarrow \mathbb{R}$ given by

$$
h=\chi_{A_{1}} \pi_{1}+\chi_{A_{2}} \pi_{2}+\chi_{A_{3}} \pi_{3},
$$

where $\chi_{A_{i}}$ is the characteristic function associated to the set $A_{i}, i=1,2,3$, and $\mathcal{F}_{1}$ is given by

$$
\mathcal{F}_{1}=\bigcup_{i, j \in\{1,2,3\}}\left[\pi_{i}^{-1}(0) \cap \pi_{j}^{-1}(0)\right]
$$

Note that the set of three coordinate axes $\mathcal{F}_{1}$ is not a codimension one variety of $\mathbb{R}^{3}$. So, the Filippov convention does not apply on $\mathcal{F}_{1} \subset \Sigma$ directly.

Moreover, let $X_{k}: \mathbb{R}^{3} \longrightarrow \mathbb{R}^{3}$ be $C^{r}$-vector fields, $k=1, \ldots, 8$ and designate by $\mathfrak{X}^{r}$ the space of $C^{r}$-vector fields on $\mathbb{R}^{3}$ endowed with the $C^{r}$-topology with $r=\infty$ or $r \geq 1$ large enough for our purposes.

In order to classify the points on $\Sigma$, for each vector field $X_{i}, i=1, \ldots, 8$, we denote $X_{i} h(p)=$ $\left\langle X_{i}(p), \nabla h(p)\right\rangle$ and $\left(X_{i}^{n} h(p)=\left\langle X_{i}(p), \nabla X_{i}^{n-1} h(p)\right\rangle\right.$, the Lie's derivatives, where $\langle\cdot, \cdot\rangle$ denote the canonical inner product. We may consider $\Omega^{r}$ endowed with the product topology and denote any element in $\Omega^{r}$ by $Z=\left(X_{1}, \ldots, X_{8}\right)$, which we will accept to be multivalued in points of $\Sigma$. The basic results of differential equations, in this context, were stated by Filippov in Filippov (1988). Related theories can be found in di Bernardo et al. (2008); Orlov (2009); Teixeira (2008) and references therein.

On $\Sigma_{i j} \backslash \mathcal{F}_{1}$ we generically distinguish three regions according to the Filippov's convention (see Filippov, 1988):

- Sewing Region: $\Sigma_{i j}^{c}=\left\{p \in \Sigma_{i j} \mid X_{i} h(p) X_{j} h(p)>0\right\}$.

- Sliding Region: $\Sigma_{i j}^{s}=\left\{p \in \Sigma_{i j} \mid X_{i} h(p)<0\right.$ and $\left.X_{j} h(p)>0\right\}$.

- Escaping Region: $\Sigma_{i j}^{e}=\left\{p \in \Sigma_{i j} \mid X_{i} h(p)>0\right.$ and $\left.X_{j} h(p)<0\right\}$.

We stress that for those points $q \in \Sigma_{i j}^{s} \cup \Sigma_{i j}^{e}$, it was established by Filippov (see Filippov, 1988) a manner to induce a vector field $Z^{s}$ on $\Sigma_{i j}$ which is tangent to $\Sigma_{i j}^{s} \cup \Sigma_{i j}^{e}$ and writes

$$
Z_{i j}^{s}=\frac{1}{\left\langle\left(X_{j}-X_{i}\right), \nabla h\right\rangle}\left[\left\langle X_{j}, \nabla h\right\rangle X_{i}-\left\langle X_{i}, \nabla h\right\rangle X_{j}\right]=\frac{X_{j} h X_{i}-X_{i} h X_{j}}{X_{j} h-X_{i} h} .
$$

In this work, the vector field $Z_{i j}^{s}$ will be called 'sliding vector field' or 'Filippov vector field'.

By a time rescaling, the sliding vector field $Z_{i j}^{s}$ is orbitally equivalent on $\Sigma_{i j}^{s}$ to the "normalized sliding vector field'

$$
\widetilde{Z}_{i j}^{s}=\left[\left\langle X_{j}, \nabla h\right\rangle X_{i}-\left\langle X_{i}, \nabla h\right\rangle X_{j}\right]=X_{j} h X_{i}-X_{i} h X_{j}
$$

Moreover, since the denominator in (2.1) could be negative on $\Sigma_{i j}^{e}$, we get that $\widetilde{Z}_{i j}^{s}$ is orbitally equivalent to $-Z_{i j}^{s}$ on $\Sigma_{i j}^{e}$.

Note that $\widetilde{Z}_{i j}^{s}$ can be $C^{r}$ extended beyond the boundary of $\Sigma_{i j}^{s} \cup \Sigma_{i j}^{e}$.

In this article, we deal with the following notion of flow. 
DeFInITION 2 The flow of $Z=\left(X_{1}, \ldots, X_{8}\right) \in \Omega^{r}$ is obtained by the concatenation of flows of $X_{1}, \ldots, X_{8}$ or $X_{i j}^{s}, i, j \in\{1, \ldots, 8\}$.

Let $p$ be a sewing point contained in $\Sigma_{j_{1}, k_{1}}$. In our approach, we allow the trajectory of $X_{i}$ by $p \in \Sigma_{j_{1}, k_{1}}$ to hit $\Sigma_{j_{2}, k_{2}}$ with $\left\{j_{1}, k_{1}\right\} \neq\left\{j_{2}, k_{2}\right\}$ after a finite time. Let $t_{1}(p)>0$ be the $X_{i}$-'flight time' employed in such journey. So we define the 'return map associated to $X_{i}^{\prime}$ ' by $\varphi_{Z_{i}}(p)=\phi_{Z_{i}}\left(t_{1}(p), p\right)=p_{1} \in \Sigma_{j_{2}, k_{2}}$. When it is possible, i.e. $p_{1}$ is still a sewing point, we repeat this procedure for it. Assuming that the trajectory which started in $p_{0}$ hits only sewing point, the 'complete return map' associated to $Z_{0}$, given by (1.1), is defined by the composition of these return maps, i.e.

$$
\varphi_{Z_{0}}(p)=\varphi_{Z_{8}} \circ \ldots \circ \varphi_{Z_{1}}(p)
$$

The starting vector field $X_{1}$ can be changed by any other $X_{j}, j=2, \ldots, 8$ since the orientation is preserved.

Alternatively, inspired in the previous ideas, if the starting point $p$ belongs to a sliding or escaping region, one can still define a complete return map. Indeed, in the next section, we construct such a map for the region $S$, since the results throughout this article address this region. The construction of such maps provide an important object in order to study the behaviour of $Z_{0}$ around the origin. The proofs of the main results require a detailed analysis of the complete return map.

In this article, we study smooth nonlinear perturbations of the model (1.1) and a complete picture of its dynamics is exhibited. It is worth to say that some constructions and ideas of Buzzi et al. (2014) are very useful in our approach.

\section{Auxiliary results}

In this section, we describe some important features about the PSVF expressed by Equation (1.1). Indeed, in Subsection 3.1 we describe its behaviour in $\Sigma$ and the sliding vector field associated to (1.1) (Lemmas A and B). Then, we study the sliding region $S$ of $\Sigma$ and the asymptotical stability at origin of an one-parameter family of perturbation of $Z_{0}$. Later on we analyse the way in which the trajectories converge to a limit set. This last analysis permits us to detect a global attractor for the trajectories of (1.1).

\subsection{Properties of System $Z_{0}$ given by (1.1)}

In this subsection, we analyse the region of discontinuity $\Sigma$. Indeed, the behaviour of $Z_{0}$ in each component of $\Sigma$ is presented by the following two lemmas.

Lemma A. The set $\Sigma \backslash S$ is contained in $\Sigma^{c}$.

Proof. It is not difficult to verify that $\Sigma \backslash S$ coincides with the sewing region. For instance, if $p=$ $(x, 0, z) \in \Sigma_{2,3}$ then $h(p)=\pi_{2}(p)$ and consequently

$$
X_{2} \pi_{2}(p) \cdot X_{3} \pi_{2}(p)=\langle(1,-1,3),(0,1,0)\rangle \cdot\langle(1,-1,1),(0,1,0)\rangle=1>0 .
$$

The other cases are analogous. 
LEMmA B. The set $S=\Sigma_{1,2} \cup \Sigma_{1,4} \cup \Sigma_{1,5}$ is the sliding region $\Sigma^{s}$ and the respective sliding vectors fields $Z_{12}^{s}, Z_{14}^{s}$ and $Z_{15}^{s}$ are given by

$$
\begin{aligned}
& Z_{12}^{s}(x, y, z)=(0,-1,1), \\
& Z_{14}^{s}(x, y, z)=(1,0,-1), \\
& Z_{15}^{s}(x, y, z)=(-1,1,0) .
\end{aligned}
$$

Proof. The proof of Lemma B is straightforward following Lemma A and using an algebraic manipulation of the formula (2.1).

Now we define a complete return map associated to the sliding vector fields of $Z_{0}$ given by (1.1). Indeed, consider the regions

$$
\begin{aligned}
& S_{1}=\Sigma_{1,2} \cap \Sigma_{1,5}, \\
& S_{2}=\Sigma_{1,2} \cap \Sigma_{1,4}, \\
& S_{3}=\Sigma_{1,4} \cap \Sigma_{1,5},
\end{aligned}
$$

which are transversal sections of the flows $Z_{12}^{s}, Z_{14}^{s}, Z_{15}^{s}$.

Given a point $s_{1} \in S_{1}$, consider the transition map $P_{1}: S_{1} \rightarrow S_{2}$ such that $P_{1}\left(s_{1}\right)=s_{2}$ is the point of $S_{2}$ where the $Z_{12}^{s}$-trajectory passing through $s_{1}$ intersects $S_{2}$. Analogously, define the transition maps $P_{2}: S_{2} \rightarrow S_{3}$ and $P_{3}: S_{3} \rightarrow S_{1}$ using the flows of $Z_{14}^{s}$ and $Z_{15}^{s}$ respectively.

Consequently the complete return map for the sliding region writes

$$
\varphi_{Z_{0}}=P_{3} \circ P_{2} \circ P_{1}: S_{1} \rightarrow S_{1}
$$

REMARK 1 In our particular case, the forward trajectory (which is the interesting situation here) has a unique choice, i.e. the forward trajectory of a point in $\mathcal{F}_{1}$ is well defined and it does not depart from the set $S$.

The following Propositions A and B describe the behaviour on $S$.

Proposition A. The set $S$ is fulfilled with periodic trajectories of (1.1).

Proof. We make use of the first return map in order to guarantee that any trajectory through a point in $S=\Sigma_{1,2} \cup \Sigma_{1,4} \cup \Sigma_{1,5}$ is closed. Indeed, consider $S_{1}$ the region of return of the trajectories and an initial condition $\left(0, y_{0}, 0\right) \in S_{1}$.

By Lemma $\mathrm{B}$, the flows $\varphi_{t}^{1,2}, \varphi_{t}^{1,4}$ and $\varphi_{t}^{1,5}$ starting in $p_{1,2}^{0}=\left(0, y_{0}, z_{0}\right), p_{1,4}^{0}=\left(x_{0}, 0, z_{0}\right)$ and $p_{1,5}^{0}=\left(x_{0}, y_{0}, 0\right)$ associated to the sliding vector fields $Z_{12}^{s}, Z_{14}^{s}$ and $Z_{15}^{s}$, respectively, are

$$
\begin{aligned}
& \varphi_{t}^{1,2}\left(p_{12}^{0}\right)=\left(0,-t+y_{0}, t+z_{0}\right), \\
& \varphi_{t}^{1,4}\left(p_{14}^{0}\right)=\left(t+x_{0}, 0,-t+z_{0}\right), \\
& \varphi_{t}^{1,5}\left(p_{15}^{0}\right)=\left(-t+x_{0}, t+y_{0}, 0\right) .
\end{aligned}
$$




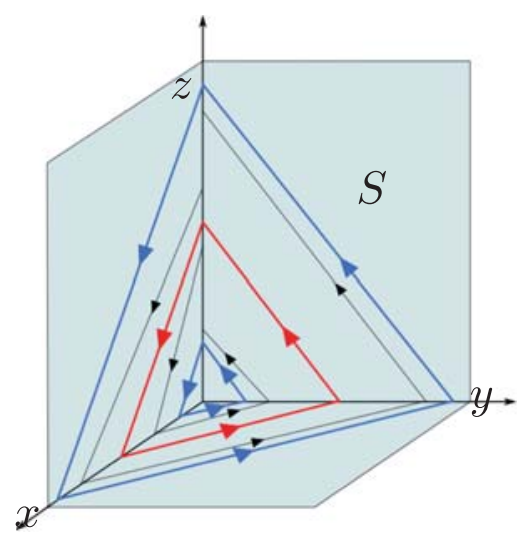

FIG. 2. $S$ is fulfilled by periodic trajectories.

Now, once $S_{1}=\Sigma_{1,2} \cap \Sigma_{1,5}$ and every future trajectory reaches $S_{1}$ transversally for positive time, the trajectory enters in $\Sigma_{1,2}$ and consequently is governed by the flow $\varphi_{t}^{1,2}$. Nevertheless, considering $p_{0}=\left(0, y_{0}, 0\right) \in S_{1}$ we obtain $\varphi_{t}^{1,2}\left(p_{0}\right)=\left(0,-t+y_{0}, t\right)$ and consequently $P_{1}\left(p_{0}\right)=\varphi_{t=y_{0}}^{1,2}\left(p_{0}\right)=$ $\left(0,0, y_{0}\right) \in S_{2}$.

From the point $\left(0,0, y_{0}\right)$ the trajectory enters in $\Sigma_{1,4}$ through the flow $\varphi_{t}^{1,4}$ and we get $\varphi_{t}^{1,4}\left(0,0, y_{0}\right)=$ $\left(t, 0,-t+y_{0}\right)$. Again, taking $t=y_{0}$, we have $P_{2}\left(0,0, y_{0}\right)=\varphi_{t=y_{0}}^{1,4}\left(0,0, y_{0}\right)=\left(y_{0}, 0,0\right) \in S_{3}$. Finally, the flow $\varphi_{t}^{1,5}$ starting at the point $\left(y_{0}, 0,0\right)$ is $\varphi_{t}^{1,5}\left(y_{0}, 0,0\right)=\left(-t+y_{0}, t, 0\right)$, then $P_{3}\left(y_{0}, 0,0\right)=$ $\varphi_{t=y_{0}}^{1,5}\left(y_{0}, 0,0\right)=\left(0, y_{0}, 0\right)=p_{0} \in S_{1}$, i.e. the first return map $\varphi_{Z_{0}}: S_{1} \rightarrow S_{1}$ is given by

$$
\varphi_{Z_{0}}\left(y_{0}\right)=\varphi_{t=y_{0}}^{1,5} \circ \varphi_{t=y_{0}}^{1,4} \circ \varphi_{t=y_{0}}^{1,2}\left(y_{0}\right)=y_{0} .
$$

Therefore, through every point $p_{0}=\left(0, y_{0}, 0\right) \in S_{1}$ passes a periodic trajectory, which means that $S=\Sigma_{1,2} \cup \Sigma_{1,4} \cup \Sigma_{1,5}$ is fulfilled with periodic orbits.

Figures 2 and 3 illustrate the situation described in Proposition A. As we can see, the origin of the system of coordinates looks like a center once every neighbourhood of it in $S$ is fulfilled with periodic trajectories. Moreover, Proposition B tells that this continuum of periodic trajectories is a global attractor of System (1.1).

The next proposition tell us that every trajectory of $Z_{0}$ in $\mathbb{R}^{3}$ converges to $S$.

Proposition B. The set $S$ is a global attractor for $Z_{0}$.

Proof. We start considering the trivial case, i.e. taking a point $q \in S=\Sigma_{1,2} \cup \Sigma_{1,4} \cup \Sigma_{1,5}$. Then, by Proposition $\mathrm{A}$, the $Z_{0}$-trajectory through $q$ is periodic and we get the result in this case. Now, consider a point $p_{1}=\left(x_{1}, y_{1}, z_{1}\right) \in R_{1}$ and $\varphi_{t}^{1}\left(p_{1}\right)$ the flow associated to the vector field $Z_{1}$. Clearly $\varphi_{t}^{1}\left(p_{1}\right)=\left(-t+x_{1},-t+y_{1},-t+z_{1}\right)$. Thus, taking $t_{1}=\min \left\{x_{1}, y_{1}, z_{1}\right\}$ it holds $\varphi_{t_{1}}^{1}\left(p_{1}\right)=q \in S$ which is periodic, so the result is true.

Now we consider a point $p$ in $\bigcup_{i=2,{ }_{8}} R_{i}$ and we must prove that $p$ converges to $S$. Indeed, without loss of generality, take $p=p_{6} \in \Sigma_{5,6} \backslash S$ since all points in $R_{5}$ reaches $\Sigma_{5,6}$. Observe that the trajectory $\varphi_{t}^{6}$ through $p_{6}=\left(x_{6}, y_{6}, z_{6}\right)$ writes $\varphi_{t}^{6}\left(p_{6}\right)=\left(-t+x_{6}, t+y_{6}, t+z_{6}\right)$. Once $y_{6}>0$ and $t+y_{6}$ is increasing 


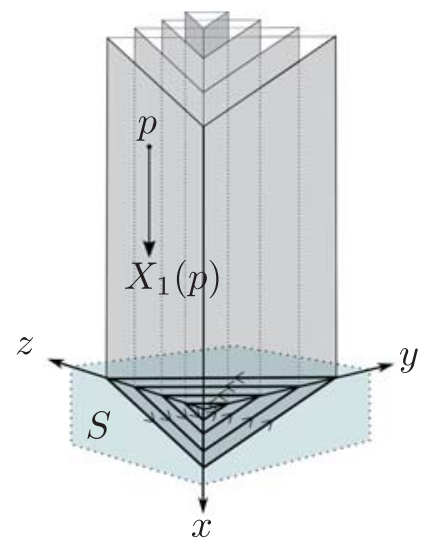

FIG. 3. The trajectories going to the 3D triangular center.

in $t$ we get $\varphi_{t}^{6}\left(p_{6}\right) \cap \Sigma_{6,7}=\emptyset$. Consequently, each point starting in $\Sigma_{5,6}$ reaches $\Sigma_{2,6}$ in a finite time in sewings points and enters in the region $R_{2}$ where the dynamics is governed by the flow associated to this region, namely, $\varphi_{t}^{2}$.

On the region $R_{2}$, the flow starting on a point $p_{2}=\left(x_{2}, y_{2}, 0\right) \in \Sigma_{2,6}$ is given by $\varphi_{t}^{2}\left(p_{2}\right)=\left(t+x_{2},-t+\right.$ $y_{2}, 3 t$ ), where $x_{2}<0$ and $y_{2}>0$. Note that the trajectory through $p_{2}$ meets $\Sigma_{1,2}$ when $t+x_{2}=0$, i.e. for $t=-x_{2}>0$. On the other hand, if $t=y_{2}$, then $\varphi_{t=y_{2}}^{2}\left(p_{2}\right) \in \Sigma_{2,3}$. Consequently, the plane $\Gamma_{2}=\left\{(x, y, z) \in \mathbb{R}^{3} \mid y=-x, x<0, y>0\right\}$ separates the region $R_{2}$ into two disjoint parts, one of them having the set $S$ as it $\omega$-limit and the other one reaching the set $\Sigma_{2,3}$.

Those points which reach $\Sigma_{2,3}$ have a behaviour similar to the points of $\Sigma_{5,6}$. In fact, all they go to $\Sigma_{3,4}$ and the plane $\Gamma_{4}=\left\{(x, y, z) \in \mathbb{R}^{3} \mid z=-y, y<0, z>0\right\}$ separates the region $R_{4}$ into two disjoint parts, one of them having the set $S$ as it $\omega$-limit and the other one reaching the set $\Sigma_{4,8}$.

Again, those points which reach $\Sigma_{4,8}$ has a behaviour similar to the points of $\Sigma_{5,6}$ and $\Sigma_{2,3}$. In fact, all they go to $\Sigma_{5,8}$ and the plane $\Gamma_{5}=\left\{(x, y, z) \in \mathbb{R}^{3} \mid z=-x, z<0, x>0\right\}$ separates the region $R_{5}$ into two disjoint parts, one of them having the set $S$ as it $\omega$-limit and the other one reaching the set $\Sigma_{5,6}$.

Observe that each one of the planes $\Gamma_{2}, \Gamma_{4}$ and $\Gamma_{5}$ guides half of the points in $\Sigma_{2,6}, \Sigma_{3,4}$ and $\Sigma_{5,8}$, respectively, until $S$. In this way from the domain $\Sigma_{5,6}$ of the complete first return map, just the points of the region $\Phi_{1}=\left\{(0, y, z) \in \Sigma_{5,6} \mid 0<y<-z / 8\right\}$ return to $\Sigma_{5,6}$. In fact, saturating by the flow the straight line $r_{1}=\left\{(0, y, z) \in \Sigma_{5,6} \mid y=-z / 8\right\}$ we obtain that it goes to the straight line $r_{2}=\Sigma_{5,8} \cap \Gamma_{5}$.

Repeating the previous argument, we get that the complete first return map $\varphi_{Z_{0}}$ satisfies $\varphi_{Z_{0}}^{2}\left(\Phi_{1} \backslash \Phi_{2}\right) \rightarrow S$, where $\Phi_{2}=\left\{(0, y, z) \in \Sigma_{5,6} \mid 0<y<-z / 64\right\}$. Recursively, $\varphi_{Z_{0}}^{n}\left(\Phi_{n-1} \backslash \Phi_{n}\right) \rightarrow S$, where $\Phi_{n}=\left\{(0, y, z) \in \Sigma_{5,6} \mid 0<y<-z / 8^{n}\right\}$. Therefore, the result follows.

Corollary 1 Given a point in $q \in \mathbb{R}^{3}$ it is possible to determine the number of times that the trajectory through $q$ hits a connect component of $\Sigma$ before reaches $S$.

Proof. We write the proof for one connect component of $\Sigma$, e.g. $\Sigma_{5,6}$. The proof for the others components is similar. Given a point $q \in \mathbb{R}^{3}$, let $q_{1}=\left(0, y_{1}, z_{1}\right)$ the intersection of its trajectory with $\Sigma_{5,6}$. Following the final steps of the proof of the previous proposition we can see that if $\left|y_{1}\right| /\left|z_{1}\right|>1 / 8$ then the trajectory 
by $q$ converges to $S$ before it returns to $\Sigma_{5,6}$. Repeating the same argument, if $1 / 8>\left|y_{1}\right| /\left|z_{1}\right|>1 / 64$ then the trajectory through $q$ converges to $S$ after hits $\Sigma_{5,6}$ once.

In general, if $1 / 8^{n-1}>\left|y_{1}\right| /\left|z_{1}\right|>1 / 8^{n}$ then the trajectory through $q$ converges to $S$ before it hits $\Sigma_{5,6}$ a number $n-1$ of times.

\subsection{Asymptotic stability at the origin of a perturbation of $Z_{0}$}

In this subsection, we exhibit a perturbation $Z_{\varepsilon}^{0}$ of the system $Z_{0}$ for which the behaviour of the trajectories of the model presented in Bernard and el Kharroubi (1991) can be topologically reproduced. We stress that the model introduced in Bernard and el Kharroubi (1991) does not present asymptotical stability at the origin once the trajectories scape from the origin and goes to infinity (which happens in case $\varepsilon<0$ in Lemma 1). Besides, if $\varepsilon>0$ in Lemma 1 , we prove that the $Z_{\varepsilon}^{0}$ is asymptotically stable at origin.

Consider initially the smooth function $F_{\varepsilon}^{0}: \mathbb{R} \rightarrow \mathbb{R}$, such that

$$
F_{\varepsilon}^{0}(x)=\varepsilon x .
$$

Let be $Z_{0}$ given by (1.1) and

$$
Z_{\varepsilon}^{0}(x, y, z)=X_{i, \varepsilon}^{0}(x, y, z), \quad \text { if }(x, y, z) \in R_{i}, i=1,2, \ldots, 8,
$$

where

$$
X_{2, \varepsilon}^{0}=X_{2}+\left(0,2 \frac{\partial F_{\varepsilon}^{0}(z)}{\partial z}, 0\right)
$$

and $X_{i, \varepsilon}^{0}(x, y, z)=X_{i}(x, y, z)$ for $i=1,3,4,5,6,7,8$ are given by (1.2). Associated to the perturbation (3.3) we have the normalized sliding vector fields given by

$$
\begin{aligned}
& Z_{1,4, \varepsilon}^{0, s}(x, y, z)=Z_{1,4}^{s}(x, y, z)=(1,0,-1) \\
& Z_{1,5, \varepsilon}^{0, s}(x, y, z)=Z_{1,5}^{s}(x, y, z)=(-1,1,0) \\
& Z_{1,2, \varepsilon}^{0, s}(x, y, z)=\left(0,-1+\frac{\partial F_{\varepsilon}^{0}(z)}{\partial z}, 1\right) .
\end{aligned}
$$

So, we get that dynamics is governed by the flow $\phi_{Z_{1,2, \varepsilon}^{0, s}}$ of $Z_{1,2, \varepsilon}^{0}$ given in (3.4) is parameterized by

$$
\phi_{Z_{2, \varepsilon}^{0, s}}(t, p)=\left(x,-t+F_{\varepsilon}^{0}(t)+y, t+z\right)
$$

where $p=(x, y, z)$.

In the following, we present the result that treat of the stability of $Z_{\varepsilon}^{0}$ :

LEMMA 1 Consider the perturbation $Z_{\varepsilon}^{0}$, given in (3.2) of $Z_{0}$. We get

(a) if $\varepsilon>0$ then the origin is asymptotically stable;

(b) if $\varepsilon<0$ then the origin is asymptotically unstable. 
Proof. First of all, we prove that $S$ is invariant and a global attractor for $Z_{\varepsilon}^{0}$. In fact, we have that the sliding vector fields $Z_{1,4, \varepsilon}^{0, s}$ and $Z_{1,5, \varepsilon}^{0, s}$ coincides with the unperturbed case. Given $p \in \Sigma_{12}$, by (3.5) we get that trajectories of $Z_{\varepsilon}^{0}$ intercepts the axis $z$ (frontier of $\Sigma_{12}$ ) and then follows the trajectory of $Z_{1,4, \varepsilon}^{0, s}$, that coincides with $Z_{1,4}^{0, s}$. Therefore, we conclude that $S$ is invariant for $Z_{\varepsilon}^{0}$. The proof that $S$ is a global attractor of $Z_{\varepsilon}^{0}$ is similar to the proof of Proposition B and will be omitted.

As $S$ is invariant and a global attractor of $Z_{\varepsilon}^{0}$, to characterize the stability is necessary and sufficient to study the stability of the first return map $\varphi_{Z_{\varepsilon}^{0}}=\phi_{Z_{1,2, \varepsilon}^{0, s}} \circ \phi_{Z_{1,5, \varepsilon}^{0, s}} \circ \phi_{Z_{1,4, \varepsilon}^{0, s}}$.

We consider the transverse section on $S$, the domain of $\varphi_{Z_{\varepsilon}^{0}}$, given by $S_{2}=\{(0,0, z) ; z>0\}$.

So, we have $\phi_{Z_{1,2, \varepsilon}^{0, s}} \circ \phi_{Z_{1,5, \varepsilon}^{0, s}} \circ \phi_{Z_{1,4, \varepsilon}^{0, s}}\left(t,\left(0,0, z_{0}\right)\right)=\phi_{Z_{1,2, \varepsilon}^{0, s}}\left(t,\left(0, z_{0}, 0\right)\right)$. The first return map is given by the third coordinate of the point in $S_{2}$ provided by the intersection of the graph of function $G^{0}(z)=$ $-z+F_{\varepsilon}^{0}(z)+z_{0}$ with the half straight line $S_{2}$. Therefore, $\varphi_{Z_{\varepsilon}^{0}}$ satisfies

$$
z-\varphi_{Z_{\varepsilon}^{0}}(z)-F_{\varepsilon}^{0}\left(\varphi_{Z_{\varepsilon}^{0}}(z)\right)=0
$$

Explicitly, we get that $\varphi_{Z_{\varepsilon}^{0}}(0,0, z)=\left(0,0, \frac{z}{1+\varepsilon}\right)$. Therefore, if $\varepsilon>0$ then $\lim _{n \rightarrow \infty}\left(\varphi_{Z_{\varepsilon}^{0}}^{n}(0,0, z)\right)=(0,0,0)$ and if $\varepsilon<0$ then $\lim _{n \rightarrow-\infty}\left(\varphi_{Z_{\varepsilon}^{0}}^{n}(0,0, z)\right)=(0,0,0)$.

Proof of Theorem D. The proof is straightforward using Lemma 1.

\section{Properties of a specific perturbation of System $Z_{0}$}

\subsection{Analysis of the first return map of the perturbed vector field}

Consider the $C^{\infty}$-functions $F_{\varepsilon}^{i}(x): \mathbb{R} \rightarrow \mathbb{R}$, where $i=1,2,3$, such that

$$
\begin{aligned}
& F_{\varepsilon}^{1}(x)=\varepsilon x(\varepsilon-x)(2 \varepsilon-x) \ldots(k \varepsilon-x), \\
& F_{\varepsilon}^{2}(x)=x \sin \left(\pi \varepsilon^{2} / x\right), \\
& F_{\varepsilon}^{3}(x)=\varepsilon(x-\varepsilon)^{k+1},
\end{aligned}
$$

with $k \in \mathbb{N}$.

Lemma 2 Consider the function $F_{\varepsilon}^{1}(x)$ given by (4.1).

(a) If $\varepsilon<0$ then $F_{\varepsilon}^{1}$ does not have roots in $(0,+\infty)$.

(b) If $\varepsilon>0$ then $F_{\varepsilon}^{1}$ has exactly $k$ roots in $(0,+\infty)$ and these roots are $\{\varepsilon, 2 \varepsilon, \ldots, k \varepsilon\}$.

(c) $\frac{\partial F_{\varepsilon}^{1}}{\partial x}(j \varepsilon)=\varepsilon^{k+1}(-1)^{j-1} j !(k-j)$ ! for $j \in\{1,2, \ldots, k\}$. It means that such partial derivative at $j \varepsilon$ is positive for $j$ odd and negative for $j$ even. 
Proof. When $x>0$, by a straightforward calculation $F_{\varepsilon}^{1}(x)=0$ if, and only if, $(\varepsilon-x)(2 \varepsilon-x) \ldots(k \varepsilon-$ $x)=0$. So, the roots of $F_{\varepsilon}^{1}(x)$ in $(0,+\infty)$ are $\varepsilon, 2 \varepsilon, \ldots, k \varepsilon$. Moreover,

$$
\frac{\partial F_{\varepsilon}^{1}}{\partial x}(x)=\varepsilon(\varepsilon-x)(2 \varepsilon-x) \ldots(k \varepsilon-x)-\sum_{i=1, i \neq j}^{k} \varepsilon x(\varepsilon i-x) .
$$

Therefore,

$$
\begin{aligned}
\frac{\partial F_{\varepsilon}^{1}}{\partial x}(j \varepsilon)= & \varepsilon(j \varepsilon)(\varepsilon-j \varepsilon)(2 \varepsilon-j \varepsilon) \ldots((j-1) \varepsilon-j \varepsilon)((j+1) \varepsilon-j \varepsilon) \\
& ((j+1) \varepsilon-j \varepsilon) \ldots(k \varepsilon-j \varepsilon) \\
= & \varepsilon^{k+1}(-1)^{j-1} j !(k-j) !
\end{aligned}
$$

This proves items (b) and (c). Item (a) follows immediately.

Lemma 3 Consider the function $F_{\varepsilon}^{2}(x)$ given by expression (4.2). For $\varepsilon \neq 0$ the function $F_{\varepsilon}^{2}$ has infinitely many roots in $\left(0, \varepsilon^{2}\right)$, these roots are $\left\{\varepsilon^{2} / 2, \varepsilon^{2} / 3, \ldots\right\}$ and

$$
\frac{\partial F_{\varepsilon}^{2}}{\partial x}\left(\varepsilon^{2} / j\right)=\mp \pi j \text { for } j \in\{2,3, \ldots\} .
$$

It means that such derivative at $\left(\varepsilon^{2} / j\right)$ is positive for $j$ odd and negative for $j$ even.

Proof. When $x>0$, by a straightforward calculation $F_{\varepsilon}^{i}(x)=0$ if, and only if, $\sin \left(\pi \varepsilon^{2} / x\right)=0$. So, the roots of $F_{\varepsilon}^{i}(x)$ in $\left(0, \varepsilon^{2}\right)$ are $\varepsilon^{2} / 2, \varepsilon^{2} / 3, \ldots$ Moreover,

$$
\frac{\partial F_{\varepsilon}^{2}}{\partial x}(x)=\sin \left(\frac{\pi \varepsilon^{2}}{x}\right)-x \cos \left(\frac{\pi \varepsilon^{2}}{x}\right)\left(\frac{\pi \varepsilon^{2}}{x^{2}}\right) .
$$

Therefore, $\frac{\partial F_{\varepsilon}^{2}}{\partial x}\left(\varepsilon^{2} / j\right)=\mp \pi j$.

Lemma 4 Consider $F_{\varepsilon}^{3}$ given in (4.3). Then $x=\varepsilon$ is a zero of multiplicity $k$ of equation $F_{\varepsilon}^{3}(x)=0$.

Proof. In fact, by expression (4.3) we get that $F_{\varepsilon}^{3}(\varepsilon)=\frac{\partial F_{\varepsilon}^{3}}{\partial x}(\varepsilon)=\cdots=\frac{\partial^{k} F_{\varepsilon}^{3}}{\partial x^{k}}(\varepsilon)=0$ and $\frac{\partial^{k+1} F_{\varepsilon}^{3}}{\partial x^{k+1}}(\varepsilon)=$ $(k+1) ! \varepsilon \neq 0$.

Consider $Z_{0}$ given by (1.1) and

$$
Z_{\varepsilon}^{1,2,3}(x, y, z)=X_{i, \varepsilon}^{1,2,3}(x, y, z), \quad \text { if }(x, y, z) \in R_{i}, i=1,2, \ldots, 8,
$$


where

$$
\begin{aligned}
& X_{2, \varepsilon}^{1}=X_{2}+\left(0,2 \frac{\partial F_{\varepsilon}^{1}}{\partial z}(z), 0\right) \\
& X_{2, \varepsilon}^{2}=X_{2}+\left(0,2 \frac{\partial F_{\varepsilon}^{2}}{\partial z}(z), 0\right) \\
& X_{2, \varepsilon}^{3}=X_{2}+\left(0,2 \frac{\partial F_{\varepsilon}^{3}}{\partial z}(z), 0\right)
\end{aligned}
$$

and $X_{i, \varepsilon}^{1,2,3}(x, y, z)=X_{i}(x, y, z)$ for $i=1,3,4,5,6,7,8$ are given by (1.2). Associated to the perturbation (4.5) we have the normalized sliding vector fields given by

$$
\begin{aligned}
Z_{1,4, \varepsilon}^{1,2,3, s}(x, y, z) & =Z_{1,4}^{s}(x, y, z)=(1,0,-1) \\
Z_{1,5, \varepsilon}^{1,2,3, s}(x, y, z) & =Z_{1,5}^{s}(x, y, z)=(-1,1,0) \\
Z_{1,2, \varepsilon}^{1, s}(x, y, z) & =\left(0,-1+\frac{\partial F_{\varepsilon}^{1}}{\partial z}(z), 1\right) \\
Z_{1,2, \varepsilon}^{2, s}(x, y, z) & =\left(0,-1+\frac{\partial F_{\varepsilon}^{2}}{\partial z}(z), 1\right) \\
Z_{1,2, \varepsilon}^{3, s}(x, y, z) & =\left(0,-1+\frac{\partial F_{\varepsilon}^{3}}{\partial z}(z), 1\right) .
\end{aligned}
$$

A straightforward calculation shows that the trajectories $\phi_{Z_{1,2, \varepsilon}^{i, s}}$ of $Z_{1,2, \varepsilon}^{i}$, with $i=1,2,3$, given in (4.6) are parameterized by

$$
\begin{aligned}
& \phi_{Z_{2, \varepsilon}^{1, s}}(t, p)=\left(x,-t+F_{\varepsilon}^{1}(t)+y, t+z\right) \\
& \phi_{Z_{2, \varepsilon}^{2, s}}(t, p)=\left(x,-t+F_{\varepsilon}^{2}(t)+y, t+z\right) \\
& \phi_{Z_{2, \varepsilon}^{3, s}}(t, p)=\left(x,-t+F_{\varepsilon}^{3}(t)+y, t+z\right),
\end{aligned}
$$

where $p=(x, y, z)$.

Remark 2 Take $Z_{\varepsilon}=Z_{\varepsilon}^{i}$, with $i=1,2$, 3. It is easy to see that $Z_{\varepsilon} \rightarrow Z_{0}$ when $\varepsilon \rightarrow 0$, in the $C^{r}$-topology.

The next result takes into account the first return map of the perturbed sliding vector fields. However, from now on we consider the region $S_{2}$ as starting point instead of $S_{1}$. Of course, in this case the return map can be obtained in a totally analogous way.

Lemma 5 The first return map of $\varphi_{Z_{\varepsilon}^{1,2}}: S_{2} \rightarrow S_{2}$ satisfies the equation

$$
z-\varphi_{Z_{\varepsilon}^{1,2}}(z)-F_{\varepsilon}^{1,2}\left(\varphi_{Z_{\varepsilon}^{1,2}}(z)\right)=0
$$


Proof. Consider the initial condition $p_{0}=\left(0,0, z_{0}\right) \in S_{2}$. The first return map is given by $\phi_{Z_{1,2, \varepsilon}^{1,2, s}} \circ \phi_{z_{1,5, \varepsilon}^{1,2, s}} \circ$ $\phi_{Z_{1,4, \varepsilon}^{1,2, s}}$ By expression (3.1) and (4.7), we get that $\phi_{Z_{1,2, \varepsilon}^{1,2, s}} \circ \phi_{Z_{1,5, \varepsilon}^{1,2, s}} \circ \phi_{Z_{1,4, \varepsilon}^{1,2, s}}\left(t,\left(0,0, z_{0}\right)\right)=\phi_{Z_{1,2, \varepsilon}^{1,2, s}}\left(t,\left(0, z_{0}, 0\right)\right)$. So, as we get in the proof of Proposition 1, the first return map is given by the third coordinate of the point in $S_{2}$ given by the intersection of the graph of function $G^{1,2}(z)=-z+F_{\varepsilon}^{1,2}(z)+z_{0}$ with the half straight line $S_{2}$. Therefore, $\varphi_{Z_{\varepsilon}^{1,2}}$ satisfies

$$
z-\varphi_{Z_{\varepsilon}^{1,2}}(z)-F_{\varepsilon}^{1,2}\left(\varphi_{Z_{\varepsilon}^{1,2}}(z)\right)=0
$$

LEMMA 6 The first return map $\varphi_{Z_{\varepsilon}^{3}}$ associated to $Z_{\varepsilon}^{3}$ is expressed by

$$
\varphi_{Z_{\varepsilon}^{3}}(z)=z+\varepsilon(z-\varepsilon)^{k+1}
$$

Proof. As the previous case, we get that $\varphi_{z_{\varepsilon}^{3}}(0,0, z)=P_{1} \circ P_{3} \circ P_{2}(0,0, z)=P_{1}(0, z, 0)$ and the application $\Pi_{3}$ is given implicitly as one of the solutions $z$ of

$$
G^{3}(z, \varepsilon)=0
$$

where $G^{3}(z, \varepsilon)=-z+F_{\varepsilon}^{3}(z)+y$. Note that putting $y=\varepsilon$ then $z=\varepsilon$ is a solution of (4.8). In other words, $P_{1}(0, \varepsilon, 0)=(0,0, \varepsilon)$. Considering the change of coordinates $\bar{z}=z-\varepsilon$ and $\bar{y}=y-\varepsilon$ then (4.8) becomes

$$
-\bar{z}+\varepsilon \bar{z}^{k+1}+\bar{y}=0 .
$$

Therefore, we obtain

$$
\varphi_{Z_{\varepsilon}^{3}}(0,0, z)=z-\varepsilon(z-\varepsilon)^{k+1}
$$

\subsection{Limit cycles of the perturbed vector fields $Z_{\varepsilon}^{1,2}$}

Proposition 1 Consider $Z_{\varepsilon}^{1,2}$ given by expression (4.4). Then $Z_{\varepsilon}^{1}$ has exactly $k$ limit cycles and $Z_{\varepsilon}^{2}$ has infinite many limit cycles, all of them situated in $S$. Moreover, these limit cycles are hyperbolic repeller limit cycles if $j$ is odd and a hyperbolic attractor limit cycles if $j$ is even.

Proof. The proof that $S$ is invariant and a global attractor for $Z_{\varepsilon}^{1,2}$ is analogous that we done in Proposition 1 and we omit it.

So, if there exists limit cycles, then they are situated at $S$. Moreover, when we restrict the flow of $Z_{\varepsilon}^{1,2}$ to $S$, by Proposition B, the fixed points of the first return map $\varphi_{Z_{\varepsilon}^{1,2}}=\phi_{Z_{1,2, \varepsilon}^{1,2, s}} \circ \phi_{Z_{1,5, \varepsilon}^{1,2, s}} \circ \phi_{Z_{1,4, \varepsilon}^{1,2, s}}$ provide us the periodic orbits of systems $Z_{\varepsilon}^{1,2}$. Since these fixed points are isolated (limit cycles), the stability of each one is given by the stability of the fixed point of the difeomorphism $\varphi_{Z_{\varepsilon}^{1,2}}$. In both cases, we consider the transverse section on $S$, the domain of $\varphi_{Z_{\varepsilon}^{1,2}}$, given by $S_{2}=\{(0,0, z) ; z>0\}$.

By Lemma 5, we get that the fixed points of the first return maps are given by the zeros of the function $F_{\varepsilon}^{1,2}(z)$. Considering the first perturbation $Z_{\varepsilon}^{1}$, by Lemma 2, we conclude that these roots in $S_{2}$ for $\varepsilon>0$, are given by $\varepsilon, 2 \varepsilon, \ldots, k \varepsilon$. Therefore, we get that the fixed points of $\varphi_{Z_{\varepsilon}^{1}}$ are given by $p_{1}=(0,0, \varepsilon), p_{2}=(0,0,2 \varepsilon), \ldots, p_{k}=(0,0, k \varepsilon)$ with $p_{i} \in S_{2}, i=1, \ldots, k$. 
So, the stability of these fixed points are given by item $(c)$ of Lemma 2 and provides that $p_{j}$ is a hyperbolic repeller fixed point for $j$ odd and a hyperbolic attractor fixed point for $j$ even. In this way, we conclude that the orbits $\gamma_{j}^{1}$, passing through $p_{j}$ of $Z_{\varepsilon}^{1}$ are hyperbolic repeller limit cycles if $j$ is odd and a hyperbolic attractor limit cycles if $j$ is even.

In an analogous way, considering the second perturbation $Z_{\varepsilon}^{2}$, the fixed points of $\varphi_{Z_{\varepsilon}^{2}}$, are given by the zeros of $F_{\varepsilon}^{2}$. Therefore, by Lemma 3, we get that periodic orbits of $Z_{\varepsilon}^{2}$ are given by the trajectories $\gamma_{j}^{2}$ passing through the points $q_{j}=\left(0,0, \varepsilon^{2} / j\right), j \in\{2,3, \ldots\}$. As the previous case, the stability is given by the Lemma 3. Moreover, $q_{j}$ is a hyperbolic repeller fixed point for $j$ odd and a hyperbolic attractor fixed point for $j$ even. Therefore, $\gamma_{j}^{2}$ are hyperbolic repeller limit cycles if $j$ is odd and a hyperbolic attractor limit cycles if $j$ is even.

REMARK 3 (Asymptotically stability of limit cycles) One should observe that the results of the current section also guarantee the asymptotical stability of some limit cycles. We remember that these limit cycles are obtained through the perturbation of the center contained in $S$, stated in Proposition A. Indeed, Proposition 1 assures that, by using Lemmas 4 and 5, the limit cycles coming from the perturbation of such a center are hyperbolic. Moreover, if $j$ is even, they attract every trajectories which are sufficiently close to them, i.e. those limit cycles are asymptotical stable.

\section{Proof of the main results of the article}

\subsection{Proof of Theorem $A$ and Corollary $B$}

Proof of Theorem A: Item (a): It follows from Remark 2.

Item (b): It follows from Proposition 1.

Item (c): It follows from Proposition B and a remark inside the proof of Proposition 1.

Proof of Corollary B: Suppose that the codimension of the origin of $Z_{0}$, given by (1.1) is $m<\infty$. Then, in a neighbourhood of $Z_{0}$ there are PSVFs of $m$ distinct topological types. This is a contradiction due Theorem A. So, the codimension of this singularity is infinite.

\subsection{Proof of Theorem $C$}

Let $K \subset \mathbb{R}^{3}$ be a compact set around the origin. Consider the restrictions to $K$ of $Z_{0}$ and of its perturbations. Since it will not cause confusion, we denote $\left.Z_{0}\right|_{K}=Z_{0}$ and $\left.Z_{\varepsilon}\right|_{K}=Z_{\varepsilon}$.

Let $Z_{\varepsilon}^{3}$ be the perturbation of $Z_{0}$ given by (4.4). By Lemma 6 we get that the first return map is given by

$$
\varphi_{Z_{\varepsilon}^{3}}(0,0, z)=z-\varepsilon(z-\varepsilon)^{k+1}
$$

We define the application

$$
F_{\varepsilon}^{3, j}(z)=\varepsilon(z-\varepsilon)^{j}\left(z-\frac{\varepsilon}{2}\right) \ldots\left(z-\frac{\varepsilon}{k-j}\right) .
$$


Consider $\mathcal{V}$ a neighbourhood of $Z_{0}$ and $\varepsilon>0$ sufficiently small such that $Z_{\varepsilon}^{3, j} \in \mathcal{V}$, where $Z_{\varepsilon}^{3, j}(x, y, z)=X_{i, \varepsilon}^{3, j}(x, y, z)$, if $(x, y, z) \in R_{i}, i=1,2, \ldots, 8$, with

$$
X_{2, \varepsilon}^{3, j}=X_{2}+\left(0,2 \frac{\partial F_{\varepsilon}^{3, j}}{\partial z}(z), 0\right)
$$

and $X_{i, \varepsilon}^{3, j}(x, y, z)=X_{i}(x, y, z)$ for $i=1,3,4,5,6,7,8$ are given by (1.2). Associated to the family (5.1) we have the normalized sliding vector fields given by

$$
\begin{aligned}
& Z_{1,4, \varepsilon}^{3, j, s}(x, y, z)=Z_{1,4}^{s}(x, y, z)=(1,0,-1) \\
& Z_{1,5, \varepsilon}^{3, j, s}(x, y, z)=Z_{1,5}^{s}(x, y, z)=(-1,1,0) \\
& Z_{1,2, \varepsilon}^{3, j, s}(x, y, z)=\left(0,-1+\frac{\partial F_{\varepsilon}^{3, j}}{\partial z}(z), 1\right) .
\end{aligned}
$$

A straightforward calculation shows that the trajectories $\phi_{Z_{1,2, \varepsilon}^{3, j, s}}$ of $Z_{1,2, \varepsilon}^{3, j}$ are parameterized by

$$
\phi_{Z_{1,2, \varepsilon}^{3, j, s}}(t, p)=\left(x,-t+F_{\varepsilon}^{3, j}(t)+y, t+z\right),
$$

where $p=(x, y, z)$.

The next lemma provides the expression of the first return map for $Z_{\varepsilon}^{3, j}$

LEMMA 7 The first return map of $Z_{\varepsilon}^{3, j}$ is given by

$$
\varphi_{Z_{\varepsilon}^{3, j}}(0,0, z)=z-F_{\varepsilon}^{3, j}(z) .
$$

Proof. The proof is analogous to that one presented in Lemma 6 and will be omitted.

Proof of Theorem $C$. Consider in $\Omega^{r}$ the $C^{r}$-topology with $r \geq k+1$. By Lemma 7, the expression of the first return map of $Z_{\varepsilon}^{3, j}$ is given by

$$
\varphi_{Z_{\varepsilon}^{3, j}}(0,0, z)=z-F_{\varepsilon}^{3, j}(z)
$$

and therefore the limit cycles are given by the solution of

$$
F_{\varepsilon}^{3, j}(z)=0
$$

So we conclude that $z_{1}=\varepsilon$ is a zero of multiplicity $j$ and $z_{2}=\varepsilon / 2, \ldots, z_{k-j}=\varepsilon /(k-j)$ are simple zeros of this equation, where $j=0,1, \ldots, k-1$. In this way, we obtain that for each $j$ the perturbation $Z_{\varepsilon}^{3, j}$ presenting exactly $k-j$ limit cycles.

From Proposition 21 of Buzzi et al. (2014), two PSVFs are equivalent if and only if the first return maps are conjugated. It is easy to see that the first return maps are conjugated if and only if the number of zeros are equal and with the same multiplicity $(k-j$ simple zeros and one zero of multiplicity $j$ ). So, in a neighbourhood (in the topology $C^{k+1}$ ) of $Z_{\varepsilon}^{3 . j}$ we get exactly $k$ distinctly topologically types of PSVFs, when $j=0,1, \ldots, k-1$.

Therefore, $Z_{\varepsilon}^{3}$ has codimension $k$. 


\section{Conclusion}

In this article, we consider a 3D model, given by (1.1), where eight smooth vector fields are combined, in the Filippov's sense, in order to create a center with a triangular shape at the sliding region $S$, where the sliding vector field is defined. Moreover, $S$ is a global attractor for the trajectories of (1.1).

An important remark is that all smooth vector fields considered, and also the sliding vector fields, are 'constant' and even in this case the PSVF $Z_{0}$ given by (1.1) has infinite codimension. This fact reveals the complexity involved in the analysis of piecewise smooth dynamical systems.

\section{Acknowledgements}

First of all we would like to thank the anonymous referees for so many constructive suggestions.

\section{Funding}

São Paulo Research Foundation (FAPESP) (\#2014/02134-7 to T.C.) and the CAPES (88881.030454/2013 -01 to T.C.) from the program CSF-PVE and (1576689 to T.C.) from the program PNPD ; São Paulo Research Foundation (FAPESP) ( \#2013/25828-1, \#2014/18508-3 to R.D.E.); Goiás Research Foundation (FAPEG), PROCAD/CAPES ( 88881.0 68462/2014-01, \#2012/10 267000803 to D.J.T.); CNPq/Brazil (478230/2013-3 and 443302/2014-6 to T.C. and D.J.T.); UFG as a part of project numbers 35796, 35798 and 040393.

\section{REFERENCES}

Acary, V. \& Brogliato, B. (2008) Lecture Notes in Applied and Computational Mechanics, Stuttgart: Springer. AlEXANDER, J. C. \& SEIDMAN, T. (1998) Sliding modes in intersecting switching surfaces, I: Blending, Houston $J$. Math. 24, 545-569.

BARbashin, E. A. (1970) Introduction to the Theory of Stability (T. Lukes ed) Wolters-Noordhoff Publishing, Groningen, p. 223. (Translated from the Russian by Transcripta Service, London.)

BERNARD, A. \& El KHARROUBI (1991) Régulations déterministes et stochastiques dans le premier "orthant" de $\mathbb{R}^{n}$. Stochastics Stochastics Rep., 34, 149-167.

Broucke, M. E., Pugh, C. C. \& Simić, S. N. (2001) Structural stability of piecewise smooth systems, Comput. Appl. Math., 20, 51-89.

Buzzi, C. A., DE CARvalho, T. \& TeixeIRA, M. A. (2014) Birth of limit cycles bifurcating from a nonsmooth center, Journal de Mathematiques Pures et Appliquees, 102, 36-47.

Chillingworth, D. R. J. (2002) Discontinuity geometry for an impact oscillator, Dyn. Syst., 17, 389-420.

di Bernardo, M., Budd, C. J., Champneys, A. R. \& KowalczyK, P. (2008) Piecewise-smooth Dynamical Systems - Theory and Applications. London: Springer.

DIECI, L. \& LOPEZ, L. (2011) Sliding motion on discontinuity surfaces of high co-dimension. A construction for selecting a Filippov vector field, Numerische Mathematik, 117, 779-811.

FILIPPOV, A. F. (1988) Differential Equations with Discontinuous Righthand Sides. Mathematics and its Applications (Soviet Series), Dordrecht: Kluwer Academic Publishers.

Jacquemard, A., Teixeira, M. A. \& Tonon, D. J. (2012) Piecewise smooth reversible dynamical systems at a two-fold singularity, Int. J Bifurcation Chaos, 22, 1250192-1-1250192-13.

JACQUEMARD, A., TeIXeIRA, M. A. \& TonOn, D. J. (2013) Stability conditions in piecewise smooth dynamical systems at a two-fold singularity, J. Dyn. Control Syst., 19, 47-67.

Kozlova, V. S. (1984) Roughness of a discontinuous system, Vestnik Moskovskogo Universiteta Seriya 1 Matematika Mekhanika, 5, 16-20.

Luo, C. J. (2006) Singularity and Dynamics on Discontinuous Vector Fields, Monograph Series on Nonlinear Science and Complexity, Amsterdam: Elsevier Sc. 
Llibre, J., Silva, P. R. \& Teixeira, M. A. (2015) Sliding vector fields for non-smooth dynamical systems having intersecting switching manifolds, Nonlinearity, 28, 493-507.

ORLOV, Y.V. (2009) Discontinuous Systems-Lyapunov Analysis and Robust Synthesis under Uncertainty Conditions, London: Springer.

Stewart, D. E. (2011) Dynamics with inequalities: impacts and hard constraints, Philadelphia: SIAM.

TeiXeira, M. A. (1977) Generic Bifurcation in Manifolds with Boundary, J. Differ. Equ., 25, 65-88.

TeixeIra, M. A. (1990) Stability conditions for discontinuous vector fields, J. Differ. Equ., 88, 15-29.

TeIXEIRA, M. A. (2008) Perturbation Theory for Non-smooth Systems, New York: Meyers, Encyclopedia of Complexity and Systems Science 152, 2008. 